\title{
Avaliação da fenda palpebral após aplicação de toxina botulínica tipo A em pacientes com distonias faciais
}

\author{
Evaluation of palpebral fissure after botulinum toxin type \\ $A$ injection in patients with facial dystonias
}

Mariana Eleonora Pereira Cunial ${ }^{1}$, Nilson Lopes da Fonseca Junior' ${ }^{1}$, José Ricardo Carvalho Lima Rehder ${ }^{2}$

\begin{abstract}
RESUMO
Objetivo: Avaliar a medida da fenda palpebral em pacientes com blefaroespasmo essencial benigno (BEB) e espasmo hemifacial (EHF) após a aplicação periocular de toxina botulínica tipo A. Métodos: Foram estudados pacientes portadores de BEB e EHF submetidos à injeção periocular de toxina botulínica tipo A pela técnica inner orbital de aplicação. Os pacientes foram fotografados em PPO antes da aplicação e catorze dias depois dela. A fenda palpebral foi mensurada nestas imagens por meio de processamento computadorizado de imagens, utilizando o programa ImageJ. As alterações da fenda palpebral foram observadas comparando-se as medidas obtidas no pré e pós-aplicação. Resultados: Comparando-se as imagens obtidas com o programa ImageJ, houve aumento estatisticamente significante $(\mathrm{p}<0,001)$ da fenda palpebral em 14 olhos $(51,8 \%)$ após a aplicação de injeção periocular da toxina botulínica e nenhuma das imagens analisadas apresentou diminuição da fenda palpebral. Conclusão: No presente estudo, os pacientes portadores de distonias faciais apresentaram aumento de fenda palpebral estatisticamente significante após aplicação periocular de toxina botulínica tipo A.
\end{abstract}

Descritores: Distonia facial/quimioterapia; Toxinas botulínicas tipo A/uso terapêutico; Toxina botulínica tipo A/efeitos adversos

\begin{abstract}
Purpose: To evaluate the measurement of palpebral fissure in patients with facial dystonias before and after periocular injection with botulinum toxin type A. Methods: We studied patients with benign essential blepharospasm and hemifacial spasm underwent periocular injection of botulinum toxin type A by the inner orbital technique of application. Patients were photographed 14 days before and after application. The palpebral fissure was measured in these images by means of computerized image processing using the program ImageJ. The palpebral fissure changes were observed by comparing the measurements obtained before and after application. Results: Comparing the images obtained with the program ImageJ, there was a statistically significant increase $(p<0.001)$ of the palpebral fissure in 14 eyes $(51,8 \%)$ after the application of periocular injection of botulinum toxin and the images analyzed showed no decrease of the palpebral fissure. Conclusion: In this study, patients with facial dystonias showed increased palpebral fissure periocular statistically significant after application of botulinum toxin type A.
\end{abstract}

Keywords: Facial dystonia/drug therapy; Botulinum toxin, type A/therapeutic use; Botulinum toxin/adverse effects

\footnotetext{
${ }^{1}$ Setores de Plástica Ocular, Órbita e Vias Lacrimais da Disciplina de Oftalmologia da Faculdade de Medicina do ABC (FMABC) - Santo André (SP), Brasil;

${ }^{2}$ Disciplina de Oftalmologia da Faculdade de Medicina do ABC (FMABC) - Santo André (SP), Brasil.
}

Os autores declaram não haver conflitos de interesse

Recebido para publicação em: 29/8/2011 - Aceito para publicação em: 20/6/2012

Rev Bras Oftalmol. 2012; 71 (6): 368-71 


\section{INTRODUÇãO}

D istonias faciais são caracterizadas por contrações musculares involuntárias repetitivas e frequentemente levam ao desenvolvimento de posturas anormais ${ }^{(1)}$. $\mathrm{O}$ início destas distonias faciais é insidioso, com o aumento da freqüência do piscar. O paciente acredita ser uma irritação ocular desencadeada pela luz ou diminuição do filme lacrimal e os anos podem se passar até que seja feito o diagnóstico correto ${ }^{(2)}$.

Trata-se de uma doença multifatorial em sua origem e manifestação. Alguns pacientes podem apresentar também contrações involuntárias de outros grupos musculares, caracterizando a síndrome de Meige, síndrome de Brueghel ou distonia orofacial idiopática $^{(3)}$.

Na prática oftalmológica, as distonias faciais mais frequentemente observadas são o blefaroespasmo essencial benigno $(\mathrm{BEB})$ e o espasmo hemifacial $(\mathrm{EHF})^{(4)}$.

As modalidades de tratamento dessas alterações consistem em medicações via oral, intervenções cirúrgicas, aplicação local de toxina botulínica e outros métodos complementares, como psicoterapia ${ }^{(4-6)}$. Dentre tais modalidades, a mais consagrada é a aplicação periocular de toxina botulínica tipo $\mathrm{A}^{(7)}$.

Após aplicação dessa toxina na região periocular, em alguns casos têm sido observadas complicações, como dor, edema e hematoma locais, diminuição da sensibilidade local e fotofobia, além de ectrópio e ptose palpebral ipsilaterais ${ }^{(8,9)}$.

A alteração do posicionamento palpebral é decorrente da ação desta toxina nos músculos retratores (músculo elevador da pálpebra superior, músculo de Muller, músculo frontal) ou protatores (músculos orbicular e secundários) ${ }^{(10,11)}$.

A distância entre as margens palpebrais superior e infe- rior em posição primária do olhar (PPO) tem sido utilizada para avaliar a fenda palpebral, com valores de normalidade entre 8 e $11 \mathrm{~mm}$. Se a fenda palpebral medida apresentar valor menor que $8 \mathrm{~mm}$, ptose deve ser considerada. Da mesma forma, presume-se retração palpebral se surgirem valores maiores que $11 \mathrm{~mm}^{(12)}$.

O objetivo deste estudo é avaliar a medida da fenda palpebral em pacientes com blefaroespasmo essencial benigno e espasmo hemifacial após a aplicação periocular de toxina botulínica tipo A.

\section{Métodos}

Foram estudados pacientes portadores de BEB e EHF submetidos à injeção periocular de toxina botulínica tipo A pela técnica inner orbital de aplicação (BEB totalizando 30U e EHF, 20U) no Setor de Plástica Ocular da Disciplina de Oftalmologia da Faculdade de Medicina do $\mathrm{ABC}^{(13)}$. Cada frasco desta toxina contém $100 \mathrm{U}$ de droga, devendo permanecer no congelador à temperatura de $-5^{\circ} \mathrm{C}$ até sua utilização. A droga foi reconstituída em 4,0 ml de soro fisiológico, resultando numa concentração de 2,5U da toxina em cada $0,1 \mathrm{ml}$ de solução. A diluição da toxina foi realizada alguns minutos antes da injeção. Foram injetadas 2,5U e $5 \mathrm{U}$ nos músculos protactores das pálpebras (músculo orbicular do olho, próceros e corrugadores dos supercílios), conforme demonstrado (Figura 1) ${ }^{(14)}$.

Os pacientes foram fotografados em PPO com uma câmera digital Sony Cyber-shot DSC-W370 14.1 Mega Pixels, antes da aplicação e catorze dias depois dela. A fenda palpebral foi mensurada nas imagens por meio de processamento computadorizado, utilizando-se o programa ImageJ. As imagens foram padronizadas por meio da colocação de uma etiqueta, de tamanho conhecido (13
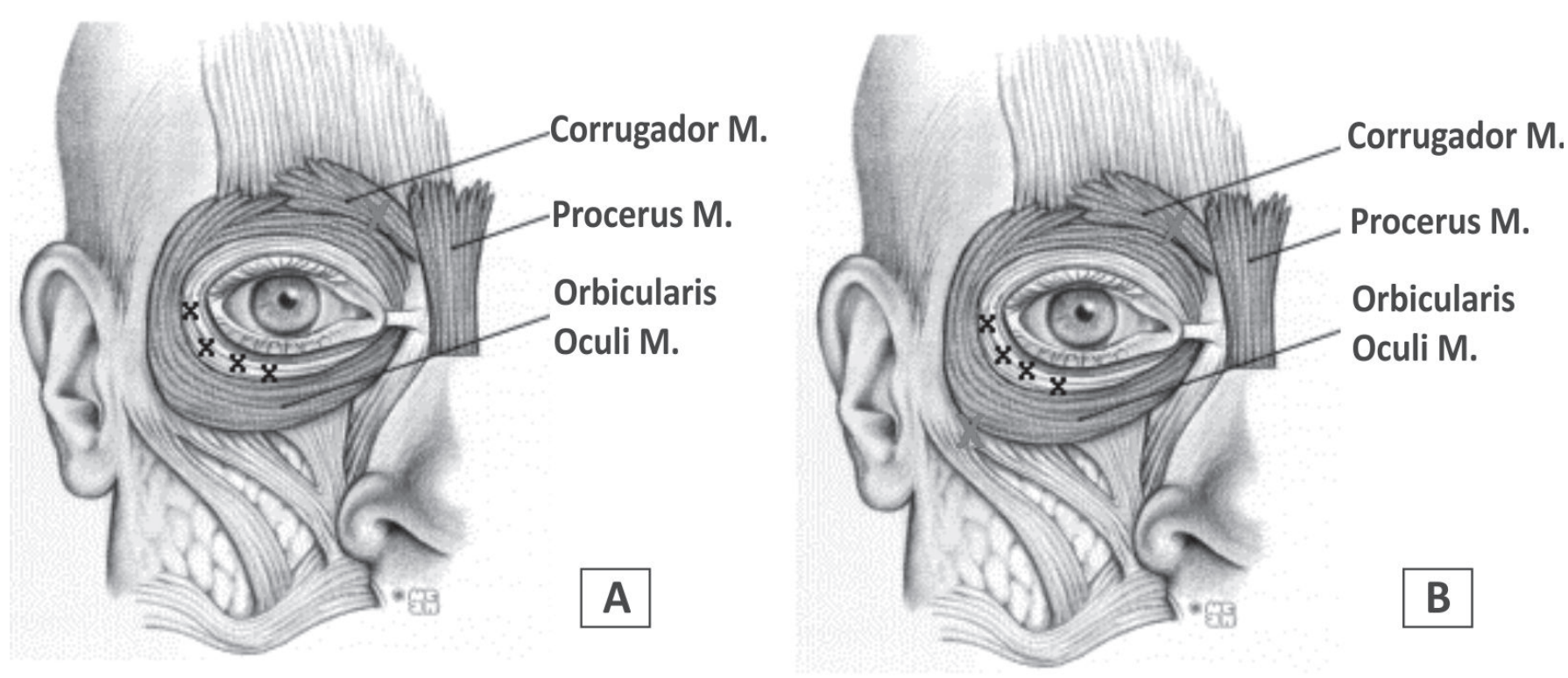

Figura 1:Técnica inner orbital de aplicação de toxina botulínica tipo A em pacientes com BEB - bilateral totalizando 30U(A) e EHF - unilateral totalizando $20 \mathrm{U}$ (B). Em destaque, os locais onde devem ser aplicadas cada injeção da toxina (ponto $\mathrm{X}=2,5 \mathrm{U}$, ponto $\mathrm{X}=5 \mathrm{U}$ ) 


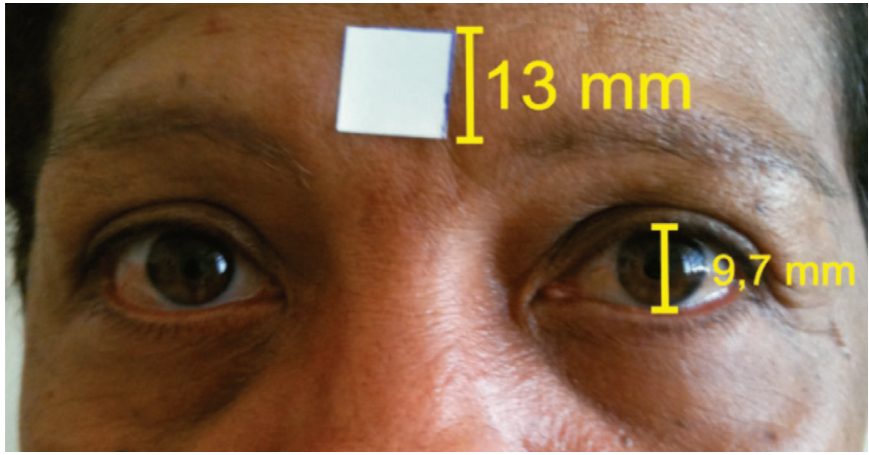

Figura 2: Método utilizado para quantificar a fenda palpebral baseado na medida da distância entre as margens palpebrais superior e inferior por meio de processamento computadorizado de imagens. A etiqueta de tamanho conhecido na fronte da paciente serve para padronizar a imagem. Nesta imagem, paciente do sexo feminino no décimo quarto dia pós aplicação de toxina botulínica tipo A para tratamento de BEB

$\mathrm{mm}$ ), na fronte dos pacientes avaliados (Figura 2).

As alterações da fenda palpebral foram observadas, comparando-se as medidas obtidas antes da aplicação e depois dela.

\section{Resultados}

Dos 19 pacientes avaliados, dez eram portadores de BEB e nove de EHF.

Comparando-se, por meio do programa ImageJ, as imagens obtidas, houve aumento da fenda palpebral em 14 olhos $(51,8 \%)$ após aplicação de injeção periocular da toxina botulínica, e nenhuma das imagens analisadas apresentou diminuição da fenda palpebral, segundo demonstrado na tabela 1 .

Neste trabalho a variável de teste $\left(\mathrm{t}_{\mathrm{n}-1}\right)$ da distribuição $t$ de Student foi de 3,724 ( $\mathrm{p}<0,001)$. Assim concluímos com 99\% de confiança que a aplicação de toxina botulínica tipo A contribuiu para o aumento da fenda palpebral dos pacientes.

\section{DısCuSSÃo}

Blefaroespasmo essencial benigno e espasmo hemifacial são distonias faciais caracterizadas por contração espontânea, espasmódica, involuntária dos músculos protatores da pálpebra (músculo orbicular, corrugador do supercílio e prócerus). A etiologia é incerta, mas há evidências de que ocorre um aumento da descarga excitatória originária dos gânglios da base ${ }^{(15)}$.

$\mathrm{O}$ uso de repetidas injeções de toxina botulínica tipo A no tratamento das distonias faciais, método de escolha em nosso Serviço, está bem estabelecido na prática médica ${ }^{(16,17)}$.A toxina botulínica tipo A inibe a liberação de acetilcolina nos terminais nervosos présinápticos por meio do bloqueio à ação do cálcio, o que resulta em denervação funcional que pode durar até seis meses ${ }^{(17)}$.

A alteração do posicionamento palpebral como complicação do tratamento de distonias faciais com injeções de toxina botulínica já foi demonstrada em outros estudos ${ }^{(8,15)}$.

Os diversos modos disponíveis para quantificar a fenda palpebral sugerem que não existe um método padrão universal. Atualmente, as medidas com uma régua, em posição primária do olhar (PPO), da fenda palpebral e da distância margem reflexo (MRD) - que corresponde à distância medida em milímetros entre a margem palpebral superior e o reflexo corneano forma-
Tabela 1

Alterações na medida da fenda palpebral nos pacientes com BEB e EHF antes e após a aplicação periocular de toxina botulínica tipo A

\begin{tabular}{|c|c|c|}
\hline Paciente & Diagnóstico & Alteração de fenda palpebral observada \\
\hline 1 & BEB & Aumento de $1,3 \mathrm{~mm}$ em OD e $2 \mathrm{~mm}$ em OE \\
\hline 2 & BEB & _ \\
\hline 3 & EHF & _ \\
\hline 4 & BEB & Aumento de $1 \mathrm{~mm}$ em AO \\
\hline 5 & BEB & - \\
\hline 6 & BEB & Aumento de $\overline{1} \mathrm{~mm}$ em AO \\
\hline 7 & EHF & Aumento de $1,6 \mathrm{~mm}$ \\
\hline 8 & BEB & _ \\
\hline 9 & EHF & - \\
\hline 10 & EHF & Aumento de $4,3 \mathrm{~mm}$ \\
\hline 11 & BEB & Aumento de $1,5 \mathrm{~mm}$ em OD e $1,2 \mathrm{~mm}$ em OE \\
\hline 12 & EHF & - \\
\hline 13 & BEB & - \\
\hline 14 & EHF & Aumento de $6,0 \mathrm{~mm}$ \\
\hline 15 & EHF & Aumento de $5,1 \mathrm{~mm}$ \\
\hline 16 & EHF & _- \\
\hline 17 & BEB & - \\
\hline 18 & EHF & - \\
\hline 19 & $\mathrm{BEB}$ & Aumento de $1,9 \mathrm{~mm}$ em OD e $2,5 \mathrm{~mm}$ em OE \\
\hline
\end{tabular}

do por uma fonte luminosa puntiforme fixada em PPO -, têm sido amplamente utilizadas ${ }^{(10,16)}$. No entanto, como a régua é um meio subjetivo, as fotografias tornam a avaliação mais precisa. $\mathrm{O}$ processamento computadorizado de imagens já foi utilizado por outros autores para analisar a fenda palpebral de pacientes com ptose congênita e avaliar o contorno palpebral em pacientes com doença de Graves ${ }^{(11,12)}$.

Os efeitos colaterais decorrentes da injeção da toxina botulínica são passageiros. Ptose palpebral é a complicação mais comum $^{(18-21)}$

Neste estudo, não houve diminuição da fenda palpebral após aplicação de toxina botulínica. Em 51,8\% dos olhos estudados, houve aumento da fenda palpebral, dado estatisticamente significante. Tais achados tornam-se mais confiáveis devido à utilização de um método preciso e objetivo de avaliação da fenda palpebral ${ }^{(10)}$.

\section{ConClusão}

Os pacientes portadores de distonias faciais apresentaram aumento de fenda palpebral estatisticamente significante após aplicação periocular de toxina botulínica tipo A.

\section{REFERÊNCIAS}

1. Hahn K, Niklai E, Garzuly F, Szupera Z. [Botulinum toxin therapy for focal dystonia]. Orv Hetil. 2009;150(29):1381-4. Hungarian.

2. Jordan DR, Patrinely JR, Anderson RL, Thiese SM. Essential blepharospasm and related dystonias. Surv Ophthalmol. 1989;34(2):123-32.

3. Anderson RL, Patel BC, Holds JB, Jordan DR. Blepharospasm: past, present, and future. Ophthal Plast Reconstr Surg. 1998;14(5):305-17. 
4. Patil B, Foss AJ. Upper lid orbicularis oculi muscle strip and sequential brow suspension with autologous fascia lata is beneficial for selected patients with essential blepharospasm. Eye (Lond). 2009;23(7):1549-53.

5. Tartaro G, Rauso R, Santagata M, Santillo V, Itro A. Lower facial contouring with botulinum toxin type A. J Craniofac Surg. 2008;19(6):1613-7.

6. Seider N, Beiran I, Miller B, Gilboa M, Kaltreider S. [Upper lid frontalis suspension as a treatment for essential blepharospasm]. Harefuah. 2005;144(11):774-6, 823. Hebrew.

7. Osawa M. [Current status and prospects of medical therapy for dystonia]. Rinsho Shinkeigaku. 2006;46(11):967-9. Japanese.

8. Roggenkämper P. [What to do in cases of inadequate effectiveness of botulinum toxin for the treatment of eyelid cramping?]. Ophthalmologe. 2007;104(9):763-6. German.

9. Carruthers J, Carruthers A. Complications of botulinum toxin type A. Facial Plast Surg Clin North Am. 2007;15(1):51-4, vi. Review.

10. Burmann TG, Valiatti FB, Correa ZM, Bayer M, Marcon I. Medida da distância reflexo margem por meio de processamento computadorizado de imagens em usuários de lentes de contato rígidas. Arq Bras Oftalmol. 2008;71(1):34-7.

11. Cruz AA, Lucchezi MC. Quantification of palpebral fissure shape in severe congenital blepharoptosis. Ophthal Plast Reconstr Surg. 1999;15(4):232-5.

12. Cruz AA, Coelho RP, Baccega A, Lucchezi MC, Souza AD, Ruiz EE. Digital image processing measurement of the upper eyelid contour in Graves disease and congenital blepharoptosis. Ophthalmology. 1998;105(5):913-8.
13. Price J, Farish S, Taylor H, O’Day J. Blepharospasm and hemifacial spasm. Randomized trial to determine the most appropriated location for botulismum toxin injections. Ophthalmology. 1997;104(5):865-8.

14. Carvalho RMLS, Gomi CF, Carvalho ALS, Matayoshi S, Moura EM. Tratamento do blefaroespasmo e distonias faciais correlatas com toxina botulínica - estudo de 16 casos. Arq Bras Oftalmol. 2003;66(1):13-6.

15. Demer JL, Holds JB, Hovis LA. Ocular movements in essential blepharospasm. Am J Ophthalmol. 1990;110(6):674-82.

16. Ceballos-Baumann A. [Botulinum toxin. Development for therapeutic purposes]. Nervenarzt. 2008;79 Suppl 1:3-8. German.

17. Badarny S, Susel Z, Honigman S. Effectivity of Dysport in patients with blepharospasm and hemifacial spasm who experienced failure with Botox. Isr Med Assoc J. 2008;10(7):520-2.

18. Barbosa ER, Haddad MS, Silva HCA, Carvalho MJ, Bittar MS. Espasmo hemifacial: tratamento com toxina botulínica. Arq Bras Neurocir. 1994;13(3):119-22.

19. Van den Bergh P, Francart J, Mourin S, Kollmann P, Laterre EC. Five-year experience in the treatment of focal movement disorders with low-dose Dysport botulinum toxin. Muscle Nerve. 1995;18(7):720-9.

20. Barbosa ER, Silva HCA, Haddad MS, Bittar MS. Blefaroespasmo: tratamento com toxina botulínica. Rev Hosp Clin Fac Med Univ São Paulo. 1996;51(6):220-3.

21. Lucci LMD. Blefaroespasmo essencial benigno. Arq Bras Oftalmol. 2002;65(5):585-9. 